\title{
Cuando la vehemencia supera la evidencia: el caso del uso de hidroxicloroquina para el tratamiento del COVID-19
}

\section{When vehemence surpasses evidence: The case of hydroxychloroquine use for the therapy of COVID-19 infection}

Correspondencia

Alonso Soto

sotosolari@gmail.com

Recibido: 22/03/2020

Arbitrado por pares

Aprobado: 24/03/2020

Citar como: Soto A. Cuando

la vehemencia supera la

evidencia: el caso del uso

de hidroxicloroquina para el

tratamiento del COVID-19. Acta

Med Peru. 2020;37(1):110-1.

doi: $h$ ttps://doi.org/10.35663/

amp.2020.371.914

\author{
Alonso Soto ${ }^{1,2}$ \\ Instituto de Investigación en Ciencias Biomédicas. Universidad Ricardo Palma. Lima, Perú \\ Departamento de Medicina. Hospital Nacional Hipólito Unanue. Lima, Perú
}

Sr Editor:

Se ha mostrado mucho entusiasmo entre la comunidad médica y las sociedades científicas con respecto al uso de la hidroxicloroquina para el manejo del COVID-19. De hecho, muchos hospitales ya están utilizando este tratamiento en pacientes hospitalizados e incluso ambulatorios sobre la base de algunos protocolos hospitalarios, guías preliminares que plantean considerar su posible uso ${ }^{[1]}$ y, particularmente, un pequeño estudio no controlado ${ }^{[2]}$ de calidad metodológica cuestionable. Al respecto, quisiera resaltar algunos puntos importantes:

A nivel internacional, las guías de la Surviving Sepsis Campaign ${ }^{[3]}$ del Hospital de Peking ${ }^{[4]}$ y una revisión publicada recientemente en la revista JAMA sobre atención de pacientes críticos con COVID-19 ${ }^{[5]}$ concluyen que no existe evidencia de que el uso de cloroquina e hidroxicloroquina brinden un beneficio clínico. A nivel nacional, el Instituto de evaluación de tecnologías sanitarias de EsSalud (IETSI) ha revisado los datos existentes hasta el momento, llegando a la misma conclusión y recalcando que en el estudio de Gautret ${ }^{[2]}$ se mostró una disminución de la excreción viral sin evaluar desenlaces clínicos (aunque en el grupo de intervención tres pacientes requirieron cuidado intensivo y uno falleció) y el grupo de controles incluyó niños, restando comparabilidad a los grupos. Aunque ningún estudio o experiencia preliminar publicada en el mundo ha demostrado eficacia clínica, hay múltiples ensayos clínicos en curso evaluando la eficacia de estas y otras drogas antivirales con el SARS-CoV-2. En todos estos documentos que incluyen una revisión extensa de la literatura, se recomienda que la evaluación del uso de cloroquina e hidroxicloroquina deben darse en el contexto de ensayos clínicos a fin de determinar su potencial eficacia ${ }^{[6]}$.

Por otro lado, ni la cloroquina ni la hidroxicloroquina son fármacos exentos de efectos adversos. Tienen el potencial de prolongar el intervalo QT y, aunque infrecuente, este efecto puede potenciarse por la interacción farmacológica -de alto riesgo- con azitromicina (https://reference.medscape.com/ drug-interactionchecker), combinación que se está recomendando indiscriminadamente sin reparar en sus peligros potenciales. Más aun, dados los casos de miocarditis, injuria miocárdica aguda y arritmias asociados al virus ${ }^{[7]}$, podría ser que esta combinación sea más dañina que beneficiosa debido al uso no regulado de la droga. Por otro lado, es importante que la comunidad no perciba este tratamiento como una cura definitiva, pues en otros países ya están apareciendo los casos de intoxicación por cloroquina frente al anuncio de su eficacia ${ }^{[8]}$. 
En estos casos de emergencia nacional e incertidumbre científica, lo que se puede considerar es el uso fuera de etiqueta, el cual se encuentra normado por la DIGEMID. La decisión personal de cada médico es respetable, pero debe informarse al paciente $y / 0$ familiares de que se trata de una terapia experimental y que se debe contar con su consentimiento. Si bien existe la posibilidad -y mucha fe- en que el uso de cloroquina o hidroxicloroquina demuestren ser útiles, también es factible que no los sean e incluso provoquen desenlaces adversos.

Finalmente, es esencial recalcar la necesidad de un proceso estandarizado pero rápido de toma de decisiones que permitan formular recomendaciones en este contexto de emergencia, respetando la institucionalidad correspondiente y desarrollando un liderazgo positivo. Cuando aparezcan terapias promisorias, es importante hacer una valoración crítica de su utilidad, sopesando los posibles beneficios y los riesgos potenciales. Aún en situaciones apremiantes, como la que atravesamos ahora, debemos recordar el primum non nocere (primero no hacer daño). La publicación de recomendaciones de manejo a cargo del Ministerio de Salud con intervención de las sociedades científicas involucradas y con actualizaciones semanales con base en la evidencia acumulada es una prioridad.

Fuente de financiamiento: Autofinanciado.

Potenciales conflictos de interés: Los autores declaran no tener conflicto de intereses.

\section{ORCID:}

Alonso Soto Tarazona, http://orcid.org/0000-0001-8648-8032

\section{REFERENCIAS BIBLIOGRÁFICAS}

1. Chinese clinical guidance for COVID-19 pneumonia diagnosis and treatment [Internet]. $7^{\circ}$ edición. China: China National Health Commission; 4 de marzo de 2020 [citado el 24 de marzo de 2020]. Disponible en http://kjfy.meetingchina.org/msite/news/show/ $\mathrm{cn} / 3337$.html.

2. Gautret P, Lagier JC, Parola P, Thuan V, Meddev L, Mailhe, et al. Hydroxychloroquine and azithromycin as a treatment of COVID-19: results of an open-label non-randomized clinical trial. Int J Antimicrob Agents. In Press 17 March 2020. doi: 10.1016/j. ijantimicag.2020.105949

3. Alhazzani W, Hylander M, Arabi YM; Loeb M, Ng M, Fan E, et al. Surviving Sepsis Campaign: Guidelines on the management of critically ill adults with coronavirus disease 2019 (COVID-19) [Internet]. Illinois: The Society of Clinical Care Medicine; 2020 [citado el 22 de marzo del 2020] Disponible en https://www.sccm.org/ getattachment/Disaster/SSC-COVID19-Critical-Care-Guidelines.pdf

4. Li T. Diagnosis and clinical management of severe acute respiratory syndrome coronavirus 2 (SARS-CoV-2) infection: an operational recommendation of Peking Union Medical College
Hospital (V2.0), Emerg Microbes Infect. 2020;9(1):582-5. doi: 10.1080/22221751.2020.1735265

5. Murthy $S$, Gomersall CD, Fowler RA. Care for critically ill patients with COVID-19. JAMA. 2020 Mar 11. doi: 10.1001/jama.2020.3633

6. Seguro Social de Salud (EsSalud). Instituto de Evaluación de Tecnologías en Salud e Investigación. Reporte breve uso de fosfato de cloroquina e hidroxicloroquina para el tratamiento de pacientes con COVID-19. Lima: EsSalud; 2020.

7. Driggin E, Madhavan MV, Bikdeli B, Chuich T, Laracy J, BondiZoccai G, et al. Cardiovascular considerations for patients, health care workers, and health systems during the coronavirus disease 2019 (COVID-19) pandemic. J Am Coll Cardiol. 2020 Mar 18. doi: 10.1016/j.jacc.2020.03.031

8. Soto A. Nigeria has chloroquine poisonings after Trump praised drug [Internet]. New York: Bloomberg LP; 21 de marzo de 2020 [actualizado el 21 de marzo de 2020, citado el 22 de marzo del 2020]. Disponible en https://www.bloomberg.com/news/ articles/2020-03-21/nigeria-reports-chloroquine-poisonings-aftertrump-praised-drug 